\title{
Resonant stripping as the origin of dwarf spheroidal galaxies
}

\section{Citation}

D’Onghia, Elena, Gurtina Besla, Thomas J. Cox, and Lars Hernquist. 2009. “Resonant Stripping as the Origin of Dwarf Spheroidal Galaxies." Nature 460 (7255): 605-7. https://doi.org/10.1038/ nature08215.

\section{Permanent link}

http://nrs.harvard.edu/urn-3:HUL.InstRepos:41381805

\section{Terms of Use}

This article was downloaded from Harvard University's DASH repository, and is made available under the terms and conditions applicable to Open Access Policy Articles, as set forth at http:// nrs.harvard.edu/urn-3:HUL.InstRepos:dash.current.terms-of-use\#OAP

\section{Share Your Story}

The Harvard community has made this article openly available.

Please share how this access benefits you. Submit a story. 


\title{
Resonant stripping as the origin of dwarf spheroidal galaxies
}

\author{
Elena D'Onghia ${ }^{1,2}$, Gurtina Besla ${ }^{2}$, Thomas J. Cox $^{2}$ \& Lars Hernquist ${ }^{2}$ \\ ${ }^{1}$ Institute for Theoretical Physics, University of Zurich, CH-8057 Zurich, Switzerland. \\ ${ }^{2}$ Harvard-Smithsonian Center for Astrophysics, 60 Garden St. MS 51, Cambridge, MA, 02138.
}

Dwarf spheroidal galaxies are the most dark matter dominated systems in the nearby Universe ${ }^{1-3}$ and their origin is one of the outstanding puzzles of how galaxies form. Dwarf spheroidals are poor in gas and stars, making them unusually faint ${ }^{4-6}$ and those known as ultra-faint dwarfs ${ }^{7,8}$ have by far the lowest measured stellar content of any galaxy. ${ }^{9,10}$ Previous theories ${ }^{11}$ require that dwarf spheroidals orbit near giant galaxies like the Milky Way, but some dwarfs have been observed in the outskirts of the Local Group. ${ }^{12}$ Here we report simulations of encounters between dwarf disk galaxies and somewhat larger objects. We find that the encounters excite a process, which we term "resonant stripping", that can transform them into dwarf spheroidals. This effect is distinct from other mechanisms proposed to form dwarf spheroidals, including mergers, ${ }^{13}$ galaxy-galaxy harassment, ${ }^{14}$ or tidal and ram pressure stripping, because it is driven by gravitational resonances. It may account for the observed properties of dwarf spheroidals in the Local Group, including their morphologies and kinematics. Resonant stripping predicts that dwarf spheroidals should form through encounters, leaving detectable long stellar streams and tails.

In the widely accepted cold dark matter theory, bound systems form hierarchically through gravitational collapse. Because gravity acts in the same manner on all forms of mass, it is expected that galaxies should contain a ratio of dark to ordinary luminous material roughly similar to the cosmic mean. However, dwarf spheroidals have much larger dark to luminous mass ratios, ${ }^{1-3}$ requiring some mechanism to efficiently separate gas and stars from dark matter in order to explain their origin. Two classes of models have been proposed to account for the properties of dwarf spheroidals. In one class, gas is either prevented from collecting in shallow potential wells of dark matter by photoheating during cosmic reionization or blown out subsequently by feedback from star formation before a significant mass in stars can accumulate. ${ }^{15,16}$ However, there are no clear signatures of reionization in the star formation histories of these galaxies, ${ }^{12}$ which in many cases 
continued to form stars beyond the reionization epoch, and for most of them the dark halo masses are likely too large $\left(10^{8}-10^{9} \mathrm{M}_{\odot}{ }^{3,9,10}\right)$ for supernova-driven winds to remove gas. ${ }^{17}$

The other class of models relies on impulsive heating by gravitational tidal forces to strip stars and reshape the stellar component of a disk-dominated dwarf galaxy. Variously referred to as "tidal stirring" 11 or "tidal shocking", ${ }^{18}$ this process operates when the gravitational potential acting on a small galaxy varies rapidly as it orbits in a larger system and can, in principle, convert a rotationally supported disk of stars into a spheroid. However, gas can radiatively dissipate energy; thus, scenarios based on gravitational heating require an additional process, i.e. ram pressure stripping, to remove the gas. ${ }^{11}$ Because gravitational heating and ram pressure stripping occur with varying efficiencies, models coupling them may rely on particular conditions to produce dwarf spheroidal galaxies.

This second class of models also requires that dwarfs orbit close to giant galaxies. Whereas several of the brightest dwarf spheroidals in the Local Group cluster around the large galaxies, some, like Cetus and Tucana, reside at great distances from both the Milky Way and Andromeda. ${ }^{6,19}$ Furthermore, many nearby dwarf spheroidals lie in the same plane as the orbits of the Magellanic Clouds and the Magellanic Stream. ${ }^{20}$ A thin planar distribution may be difficult to explain if dwarf galaxies fall in individually ${ }^{21,22}$ but can arise naturally if dwarfs fall into larger systems as members of groups of dwarfs. ${ }^{23}$ Examples of such groups have been found nearby. ${ }^{24}$ Moreover, interactions between dwarfs should be common in these environments, especially in the past when the Universe was younger and more dense.

Here we describe numerical experiments to investigate the consequences of encounters between dwarf galaxies. Figure 1 shows the time evolution of an interaction between a pair of dwarf galaxies. This could represent an interaction between dwarfs in a small group or between a dwarf and the forming Milky Way at high redshift. After 2 billion years nearly $\sim 80 \%$ of the stars are stripped away from the smaller dwarf but its surrounding dark matter halo is less strongly affected, leading to a change in the ratio of dark to luminous matter. This outcome is surprising because the stars sit at the bottom of the potential well and therefore comprise the most tightly bound material in the galaxy.

This unexpected result is caused by a gravitational process, which we term "resonant stripping". The efficient removal of stars from the smaller dwarf over the course of the interaction shown in Figure 1 is mediated by a resonance between the spin angular frequency $\left(\Omega_{s}\right)$ of its disk and the angular frequency of its orbit $\left(\Omega_{0}\right)$ around the larger dwarf. When these frequencies are comparable in magnitude and the spin and orbital angular momenta are somewhat aligned (i.e. a prograde encounter), the gravitational perturbation from the larger dwarf on a patch of the smaller dwarf's disk is always directed outwards from its center, leading to significant stripping. However, for a perfectly retrograde collision, stars in the disk are alternately pulled inward and outward with little net result. The minimal response from the dark matter is expected because these particles move on random orbits, and the net perturbation on the halo mostly averages out. 


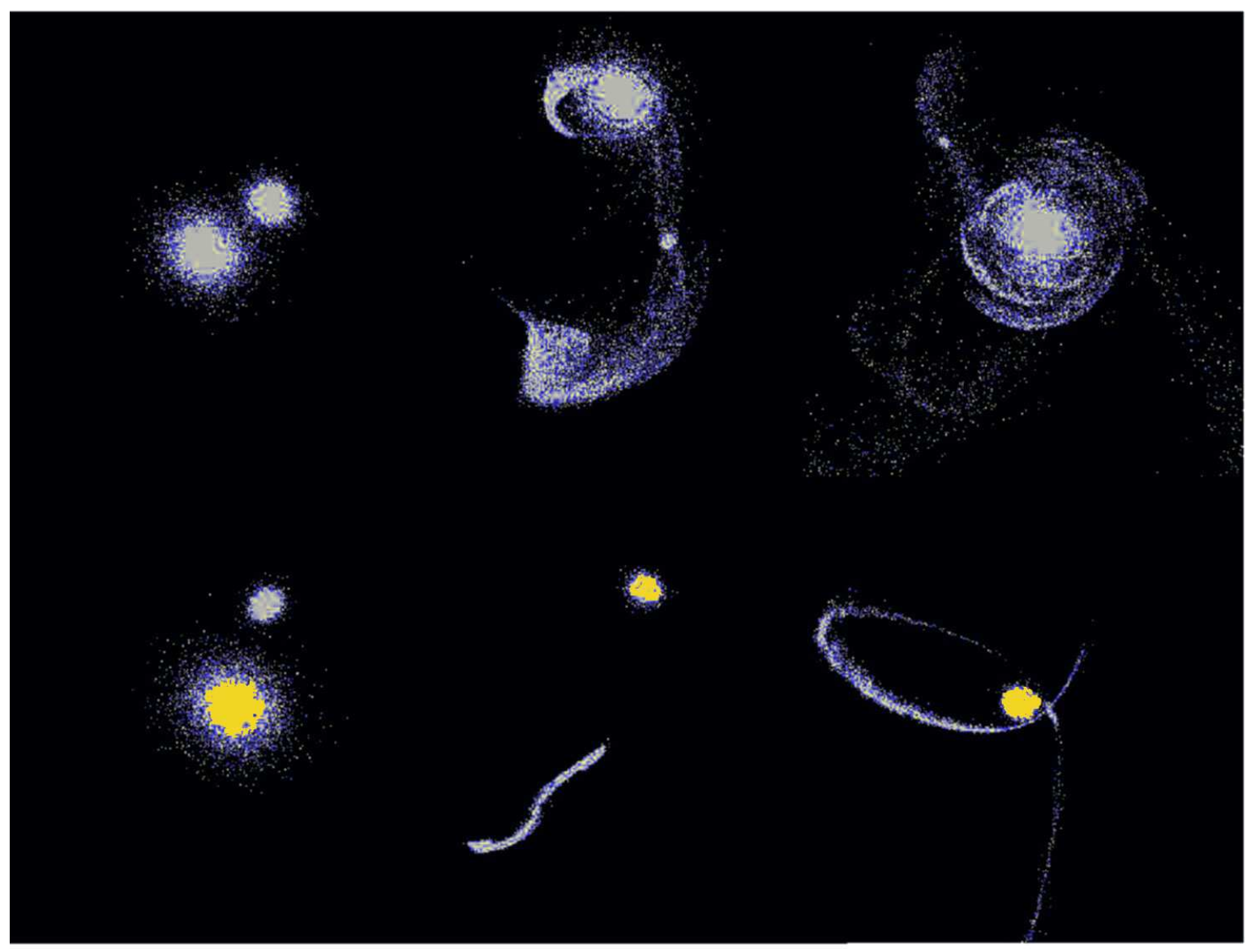

Figure 1. Encounters between galaxies. Top Row: Interaction between a dwarf galaxy with a mass of $1.7 \times 10^{8} \mathrm{M}_{\odot}$ orbiting around a larger dwarf with 100 times its mass. Only the stellar components are plotted. The upper left panel illustrates the initial set up where the two dwarfs approach one another on a somewhat prograde orbit (the disks are seen face on). The upper middle panel gives the state of the system after 2 billion years, following the first pericentric passage, and the upper right panel shows the appearance of the galaxies after 7 billion years. Each panel displays a region $100 \mathrm{kpc}$ on a side. An outcome like the one illustrated in the top panels of Figure 1 occurs preferentially when one of the interacting galaxies is between 10 and 100 times more massive than the other one. If the galaxies have nearly the same mass they will merge quickly, masking the effects of resonant stripping because nearly all the luminous matter will remain bound to the remnant. Bottom Row: Shown is the orbit of the same small galaxy (in white) around the Milky Way today (in yellow), which has 10,000 times its mass. Although the encounter is mostly prograde, the spin and orbital frequencies are no longer well-matched and the resonant response is suppressed. The left panel displays a region $150 \mathrm{kpc}$ on a side and the initial setup; the middle and right panels show an expanded view $300 \mathrm{kpc}$ on a side and give the state of the system after 2 and 7 billion years, respectively. 


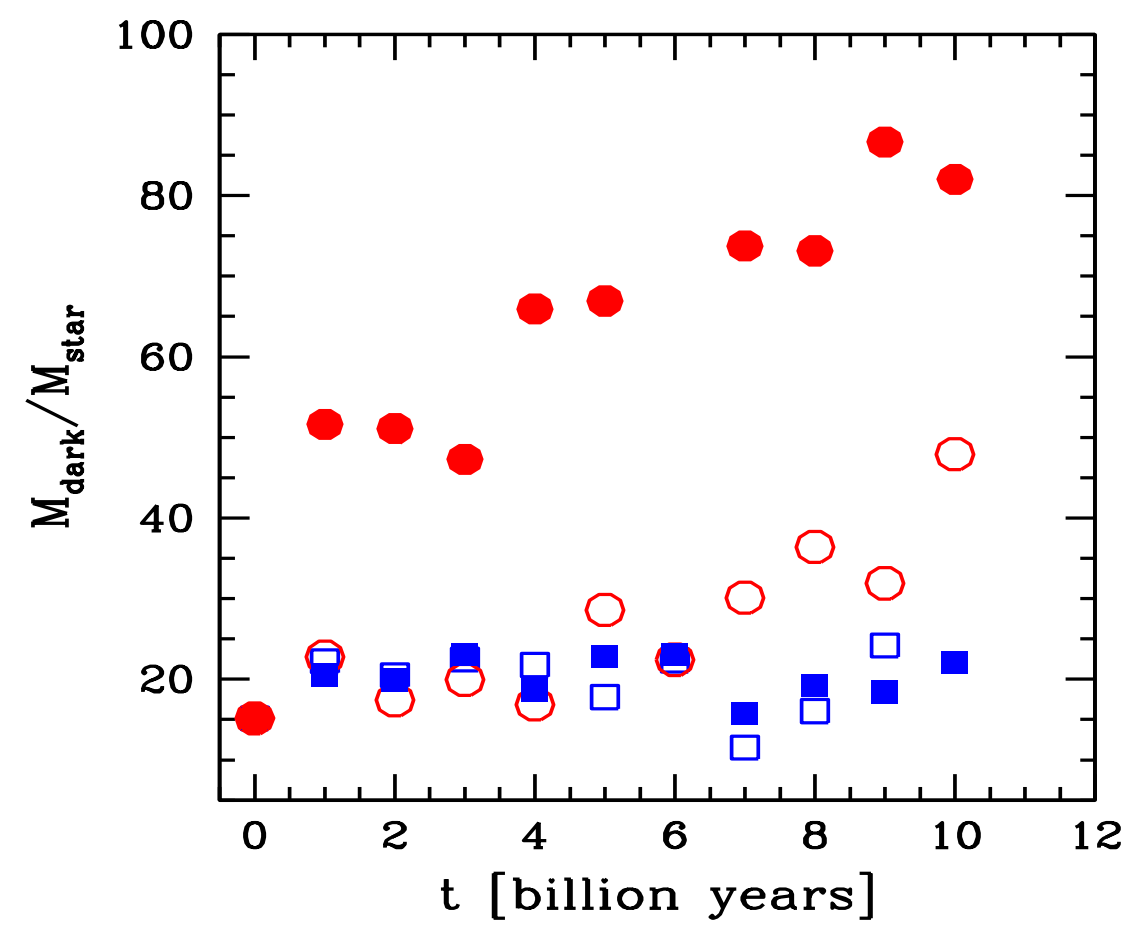

Figure 2. The time evolution of the dark-to-luminous mass ratio $\left(\mathbf{M}_{\mathrm{dm}} / \mathbf{M}_{\text {star }}\right)$. The $\mathrm{M}_{\mathrm{dm}} / \mathrm{M}_{\text {star }}$ of the smaller dwarf being resonantly stripped is computed at the tidal radius and marked by the filled red circles. The same case but for a mostly retrograde encounter is illustrated by the open red circles. A resonance occurs if $\Omega_{s}=\Omega_{0} \rightarrow \frac{v}{r} \sim \frac{V_{0}}{R_{\text {peri }}} \sqrt{(1+e)}$, where $v$ is the rotation velocity, $r$ is the size of the smaller dwarf, $V_{0}$ is the orbital velocity, $R_{\text {peri }}$ the pericentric distance, and $e$ the eccentricity of the orbit. Note that this resonance condition is not dependent on the specific choice of the pericentric distance alone but rather on the combination of the internal structure (e.g. the rotation curve) of the small dwarf and the orbital parameters. In other words, if the pericentric distance changes, the resonance condition could still be satisfied provided that the disk rotation speed were modified accordingly. If the orbit is more retrograde, stars are not preferentially removed immediately. So, after 2 billion years, the net change in $M_{d m} / M_{\text {star }}$ is a factor of 4 larger for the prograde versus retrograde cases illustrated. However, after 4 billion years the internal structure of the smaller dwarf and the orbit are affected by gravitational torques, allowing resonant stripping to occur. The ratio $\mathrm{M}_{\mathrm{dm}} / \mathrm{M}_{\mathrm{star}}$ of the small galaxy orbiting about the Milky Way today is plotted for prograde (filled blue squares) and retrograde (open blue squares) orbits. In both these cases, the spin and orbital frequencies of the galaxies are no longer comparable and the resonant interaction is suppressed, even in the prograde case. 


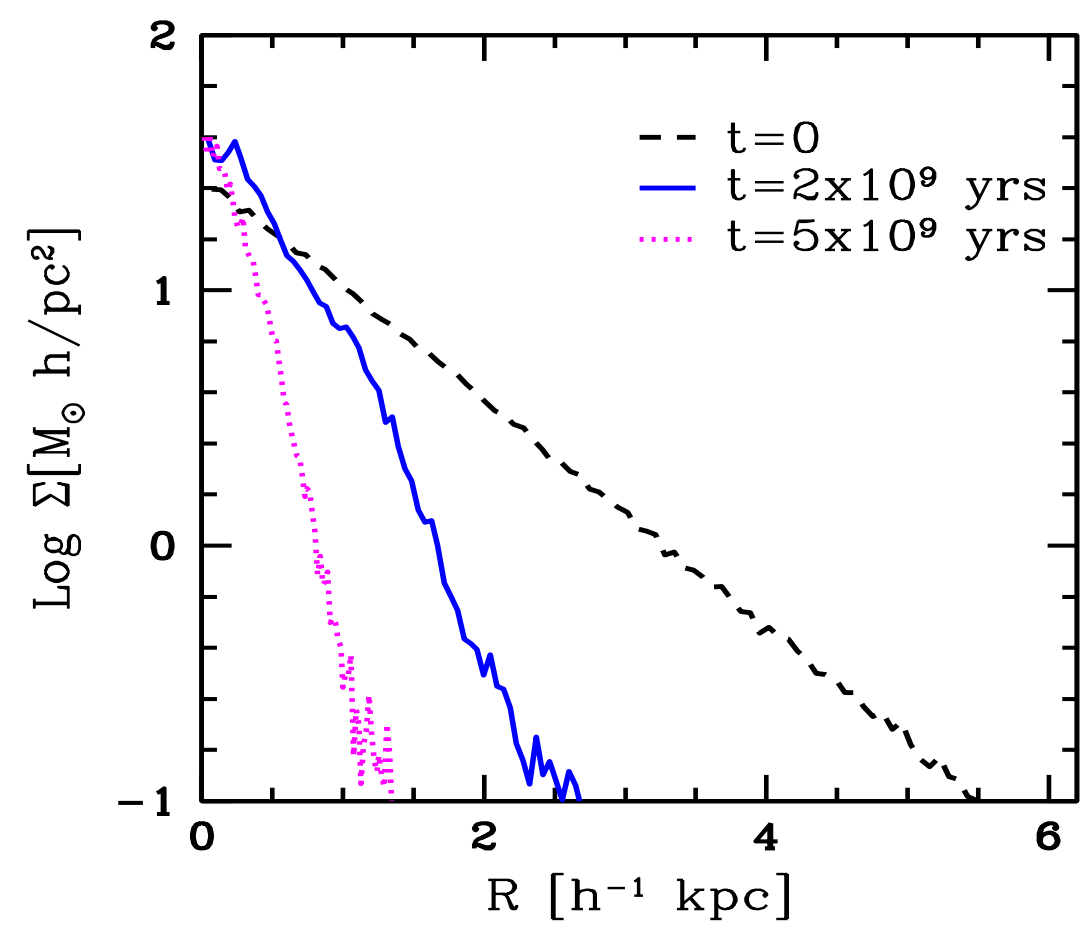

Figure 3. Radial stellar surface mass density profile of the smaller dwarf. The profile is plotted for a prograde encounter at the initial time of the simulation (dashed black line), after the first pericentric passage ( 2 billion years; solid blue line), and after 5 billion years (magenta dotted line). The profile is initially an exponential distribution with effective radius, $\mathrm{R}_{e}$, appropriate for dwarf disk galaxies. However, it evolves immediately into a more concentrated profile with $\mathrm{R}_{e} \sim 0.5 h^{-1} \mathrm{kpc}$ (blue line) and after 5 billion years the disk is converted into a compact spheroid with a smaller effective radius. 


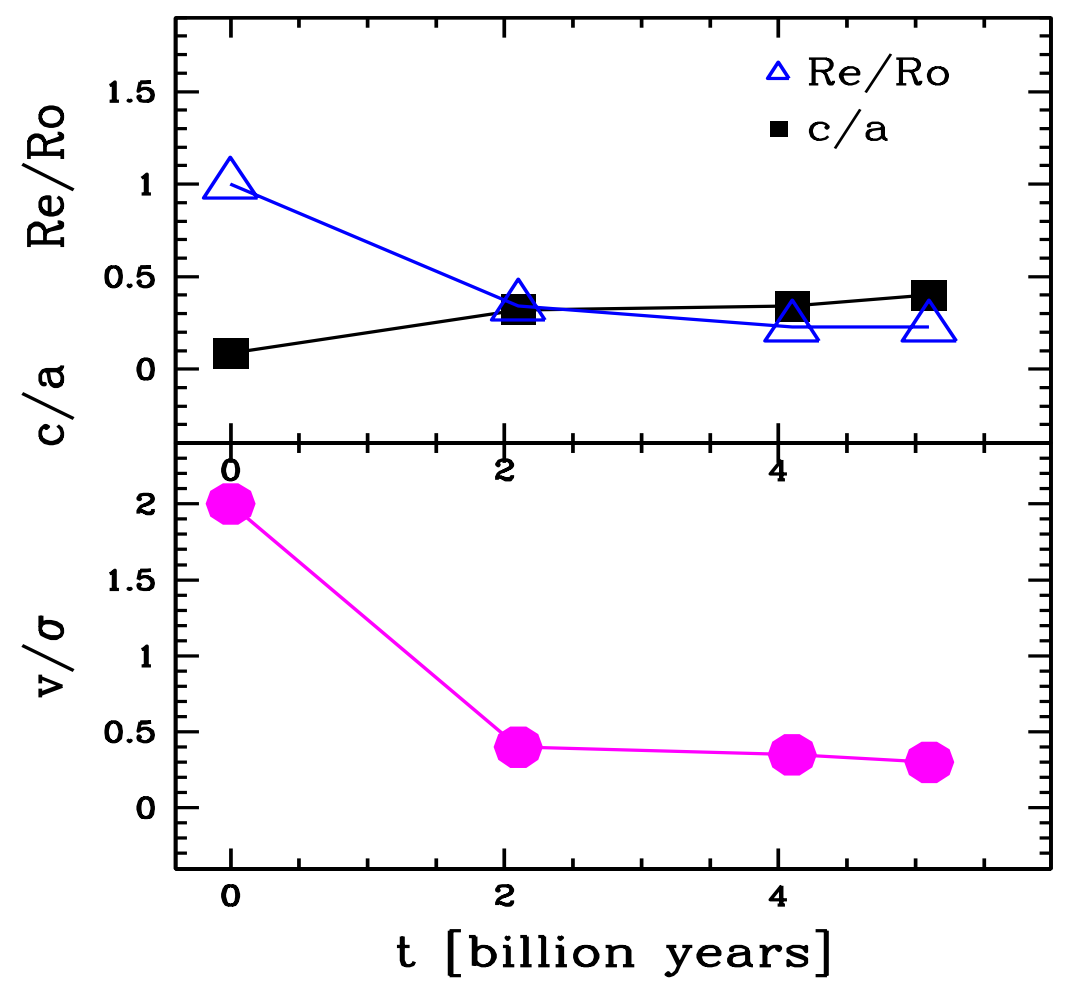

Figure 4. The time evolution of the structural properties of the small dwarf galaxy. The morphological evolution of the small dwarf galaxy over the course of the prograde encounter is quantified at characteristic times $(0,2,4$ and 5 billion years). The top panel illustrates the evolution of the effective radius, $\mathrm{R}_{e}$, normalized to its value at the beginning of the simulation and is marked by blue open triangles. The evolution of the minor to major axis ratio of the bound stellar component $(c / a)$ measured at $\mathrm{R}_{e}$ is plotted as filled squares in the same panel. The squares indicate that the initial stellar disk $(c=0)$ changes its shape immediately after 2 billion years into a more spheroidal object $(c \approx 0.5)$. This evolution owes to the complete removal of the outer stellar disk via resonant stripping. The bottom panel shows the evolution of the kinematic properties of the dwarf, measured in terms of the ratio of the centrifugal rotational velocity of the small dwarf, $v$, to its random motions, $\sigma$. The ratio $v / \sigma$ (closed circles) evolves after the first pericentric passage (2 billion years) indicating a change from a state where the rotational support at $\mathrm{R}_{e}$ is twice that from random motions to a value for $v / \sigma$ less than 0.5 - such a low value is similar to that of dwarf spheroidals not only in the Local Group but also in galaxy clusters. ${ }^{26}$ 
Although resonances have previously been invoked to produce bridges and tails in collisions between galaxies of comparable mass, ${ }^{25}$ our work shows that resonant stripping can alter the mass to light ratio of dwarfs by removing luminous material more efficiently than dark matter. In Figure 2 the luminous fraction of the smaller dwarf is plotted as a function of time during its interaction with a galaxy 100 times its mass (filled circles), compared to another simulation designed to follow a similar dwarf orbiting about the Milky Way today (filled squares). Resonant stripping is most effective for galaxies interacting on prograde orbits, as in the example shown in the top panels in Figure 1. However, our calculations further indicate that gravitational torques can alter the internal structure of the galaxies and the relative alignments of spin and orbital angular momenta, allowing the resonance to eventually act even for orbits that are initially somewhat retrograde (see open circles in Figure 2).

Resonant stripping is distinct from other processes proposed to drive galaxy evolution, such as mergers ${ }^{13}$ galaxy-galaxy harassment ${ }^{14}$ or more general heating processes, and tidal or ram pressure stripping. In particular, mechanisms that can be treated using the impulse approximation do not account for resonances because the particles in the perturbed system are assumed to remain roughly stationary over the course of the encounter. Because resonant stripping will affect gas and stars in a similar manner in a rotationally supported disk, it is simpler than models that require separate effects to strip the gas versus the stars.

Resonant stripping can drive the morphological evolution of dwarfs. When operating in low mass groups, this mechanism can pre-process dwarfs by transforming disk galaxies into spheroids before they are accreted by larger galaxies like the Milky Way. We find that dwarf spheroidal galaxies formed in this manner have properties similar to those of dwarfs observed in the Local Group. This is demonstrated in Figures 3 and 4, which show, respectively, that the final radial surface mass profiles and kinematic properties of a dwarf disk galaxy undergoing resonant stripping in our simulations are similar to those of observed dwarf spheroidals in the Local Group. ${ }^{26}$

Our model makes definite predictions that can be tested in the future. In particular, resonant stripping should be visible in situ in associations of dwarfs. Unlike in previous theories, dwarf spheroidals are thus expected to be found along with detectable stellar tails and shells, marking their formation. If this is indeed their dominant production mechanism, our model predicts that dwarf spheroidals should have similar properties in different environments, which is supported by the observed similarities between dwarf spheroidals in the Perseus cluster ${ }^{27}$ and those in the Local Group.

The efficiency of resonant stripping depends on various factors, including the internal structure of the smaller dwarf (see Supplementary Information). In the example shown in Figure 1, the interacting dwarfs were chosen to have characteristics similar to those of local dwarf disk galaxies. However, the detailed properties of young low-mass galaxies formed at early cosmic times are unknown, here we are assuming they are cold disks. If the smaller dwarf in our simulations was set up so as to satisfy the condition for resonant stripping even more precisely and over a larger portion of the disk, it is possible that more luminous 
D’Onghia et al.

material could be stripped, depleting the stellar surface density in the inner regions more significantly than in the example shown in Figure 3. In that event, resonant stripping might yield ultra-faint dwarf galaxies or even systems that are nearly entirely dark. This leads us to speculate that, when placed in a proper cosmological framework, resonant stripping might explain the missing satellite problem in the Local Group. ${ }^{28,29}$ Moreover, spectroscopic measurements indicate that the Virgo cluster is also missing satellite galaxies. ${ }^{30}$ Resonant stripping acting in low mass groups when the Universe was younger may have caused halos to lose their gas and stars, pre-processing them before these groups were accreted into galaxy clusters, providing a similar evolutionary mechanism to reproduce the luminosity functions of both galaxy groups and clusters.

Received; Accepted .

1. Kleyna, J.T., Wilkinson, M.I., Evans, N.W., Gilmore, G. \& Frayn, C. Dark matter in dwarf spheroidals - II. Observations and modeling of Draco. Mon. Not. R. Astron. Soc. 330, 778-791, (2002).

2. Chapman, S., Ibata, R., Lewis, G., Ferguson, A., Irwin, M., McConnachie, A. \& Tanvir, N. A Keck DEIMOS Kinematic Study of Andromeda IX: Dark Matter on the Smallest Galactic Scales. Astrophys. J. 632, L87-L90 (2005).

3. Wilkinson, M.I., Kleyna, J.T., Evans, N. W., Gilmore, G.F., Irwin, M.J., Grebel, E.K. Kinematically Cold Populations at Large Radii in the Draco and Ursa Minor Dwarf Spheroidal Galaxies Astrophys. J. 611, L21-L24 (2004).

4. Mateo, M. Dwarf Galaxies of the Local Group. Annual Review of Astronomy and Astrophysics. 36, 435-506 (1998).

5. Grebel, E.K. in The Stellar Content of Local Group Galaxies (eds Whitelock, P. \& Cannon, R.) 17-38, (IAU Symposium 192, ASP, San Francisco 1999).

6. Gallagher, J. S., Madsen, G. J., Reynolds, R. J., Grebel, E. K. \& Smecker-Hane, T. A. A Search for Ionized Gas in the Draco and Ursa Minor Dwarf Spheroidal Galaxies. Astrophys. J. 588, 326-330 (2003).

7. Willman, B. et al. A New Milky Way Dwarf Galaxy in Ursa Major. Astrophys. J. 626, L85-L88 (2005).

8. Zucker, D. B. et al. A New Milky Way Dwarf Satellite in Canes Venatici. Astrophys. J. 643, L103-L106 (2006).

9. Peñarrubia, J., Navarro, J.F., McConnachie, A. The Tidal Evolution of Local Group Dwarf Spheroidals. Astrophys. J. 673, 226-240 (2008).

10. Strigari, L. E., Bullock, J.S., Kaplinghat, M., Simon, J.D., Geha, M., Willman, B., Walker, M.G. A common mass scale for satellite galaxies of the Milky Way. Nature 454, 1096-1097 (2008). 
11. Mayer, L., Kazantzidis, S., Mastropietro, C., Wadsley, J. Early gas stripping as the origin of the darkest galaxies in the Universe. Nature 445, 738-740 (2007).

12. Grebel, E. K., Gallagher, J.S., III, Harbeck, D. The Progenitors of Dwarf Spheroidal Galaxies. Astrophys. J. 125, 1926-1939 (2003).

13. Toomre, A. Mergers and Some Consequences. In Evolution of Galaxies and Stellar Populations, (eds B.M. Tinsley, R.B. Larson), 401, (Conference at Yale University, 1977).

14. Moore, B., Katz, N., Lake, G., Dressler, A., Oemler, A. Galaxy harassment and the evolution of clusters of galaxies. Nature 379, 613-616 (1996).

15. Bullock, J., Kravtsov, A. \& Weinberg, D.H. Reionization and the abundance of galactic satellites. Astrophys. J. 539, 517-521 (2000).

16. Dekel, A., Silk, J. The origin of dwarf galaxies, cold dark matter, and biased galaxy formation. Astrophys. J. 303, 39-55 (1986).

17. Mac Low, M.M. \& Ferrara, A. Starburst-driven Mass Loss from Dwarf Galaxies: Efficiency and Metal Ejection. Astrophys. J. 513, 142-155 (1999).

18. Gnedin, O.Y., Hernquist, L., Ostriker, J.P. Tidal Shocking by Extended Mass Distributions Astrophys. J. 514, 109-118 (1999).

19. Sales, L., Navarro, J.F., Abadi, M.G., Steinmetz, M. Cosmic menage a trois: the origin of satellite galaxies on extreme orbits. Mon. Not. R. Astron. Soc. 379, 1475-1483 (2007).

20. Lynden-Bell, D. Dwarf galaxies and globular clusters in high velocity hydrogen streams. Mon. Not. R. Astron. Soc. 174, 695-710 (1976).

21. Libeskind, N.I., Frenk, C.S., Cole, S., Helly, J.C., Jenkins, A., Navarro, J.F., Power, C. The distribution of satellite galaxies: the great pancake. Mon. Not. R. Astron. Soc. 363, 146-152 (2005).

22. Zentner, A.R., Kravtsov, A.V., Gnedin, O.Y., Klypin, A.A. The Anisotropic Distribution of Galactic Satellites. Astrophys. J. 629, 219-232 (2005).

23. D’Onghia, E,. Lake, G. Small Dwarf Galaxies within Larger Dwarfs: Why Some Are Luminous while Most Go Dark. Astrophys. J. 686, L61-L64 (2008).

24. Tully, R.B., Rizzi, L., Dolphin, A.E., Karachentsev, I.D., Karachentseva, V.E., Makarov, D.I., Makarova, L., Sakai, S., Shaya, E.J. Associations of Dwarf Galaxies. Astronomic. J. 132, 729-748 (2006).

25. Toomre, A., Toomre, J. Galactic Bridges and Tails. Astrophys. J. 686, L61-L64 (1972).

26. Ferguson, H.C., Binggeli, B. Dwarf elliptical galaxies. Annual Review of Astronomy and Astrophysics. 6, 67-122 (1994).

27. Penny, S., Conselice, C., De Rijcke, S., Held, E. V. Hubble Space Telescope survey of the Perseus Cluster - I: The structure and dark matter content of cluster dwarf spheroidals. Mon. Not. R. Astron. Soc. 393, 1054-1062 (2009). 
28. Klypin, A., Kravtsov, A.V., Valenzuela, O., Prada, F. Where Are the Missing Galactic Satellites? Astrophys. J. 522, 82-92 (1999).

29. Moore, B., Ghigna, S., Governato, F., Lake, G., Quinn, T., Stadel, J., Tozzi, P. Dark Matter Substructure within Galactic Halos. Astrophys. J. 524, L19-L22 (1999).

30. Rines, K., Geller, M.J. Spectroscopic Determination of the Luminosity Function in the Galaxy Clusters A2199 and Virgo. Astrophys. J. 135, 1837-1848 (2008).

\section{Acknowledgements}

This research was partly supported by the EU Marie Curie Intra-European Fellowship under contract MEIF-041569 and from NSERC postgraduate fellowship. Numerical simulations were performed on the Odyssey supercomputer at Harvard University.

Author Information The authors declare that they have no competing financial interests. Correspondence and requests for materials should be addressed E.D. (edonghia@cfa.harvard.edu). 


\section{SUPPLEMENTARY INFORMATION}

This is an extension of the Letter to Nature, aimed at providing further details, in support of the results reported in the main body of the Letter.

\section{Numerical Methods}

The simulations were carried out with GADGET3, a parallel TreePM-Smoothed particle hydrodynamics (SPH) code developed to compute the evolution of stars and dark matter, which are treated as collisionless fluids. The six-dimensional phase space is discretized into fluid elements that are computationally realized as particles in the simulations. A detailed description of the code is available in the literature. ${ }^{31,32}$ Here we note its essential features.

GADGET3 is a cosmological code in which the gravitational field on large scales is calculated with a particle-mesh (PM) algorithm, while the short-range forces are computed using a tree-based hierarchical multipole expansion, resulting in an accurate and fast gravitational solver. The scheme combines the high spatial resolution and relative insensitivity to clustering of tree algorithms with the speed and accuracy of the PM method to calculate the long range gravitational field.

Pairwise particle interactions are softened with a spline kernel ${ }^{33}$ of scalelength $h_{s}$, so that they are strictly Newtonian for particles separated by more than $h_{s}$. The resulting force is roughly equivalent to traditional Plummer-softening with scalelength $\epsilon \approx h_{s} / 2.8$. For our applications the gravitational softening length is fixed to $h_{s}=70 h^{-1}$ pc throughout the evolution of the galaxy encounters. (Here, $h$ is the Hubble constant in units of 100 $\mathrm{km} / \mathrm{s} / \mathrm{Mpc}$.

At each output time, we measure the total mass of the dark halo of each galaxy using the SUBFIND algorithm. ${ }^{32}$ This code identifies as halo members particles in groups that are gravitationally self-bound and are overdense with respect to the local background.

\section{Setting the initial conditions}

Each galaxy in our study consists of a dark matter halo and a rotationally supported disk of stars. The parameters describing each component are independent and models are constructed in a manner similar to the approach described in previous works. ${ }^{34-36}$ 


\section{Dark Halo}

We model the dark matter mass distribution of each galaxy with a Hernquist ${ }^{37}$ profile:

$$
\rho_{\mathrm{dm}}=\frac{M_{\mathrm{dm}}}{2 \pi} \frac{a}{r(r+a)^{3}}
$$

with cumulative mass distribution $M(<r)=M_{\mathrm{dm}} r^{2} /(r+a)^{2}$, where $a$ is the radial scale length.

We motivate this choice for the halo profile by the fact that in its inner parts the shape of this profile is identical to the $\mathrm{NFW}^{38}$ fitting formula for the mass density distribution of dark matter halos in cosmological simulations. However, owing to its faster decline in the outer parts, the total mass described by the Hernquist model converges, allowing the construction of isolated halos without the need of an ad hoc truncation. To make the connection with common descriptions of halos in cosmological simulations we associate the Hernquist profile for the galaxy halo with a corresponding NFW-halo profile such that the inner density profiles are equivalent at radii less than the virial radius, $\mathrm{r}_{200}$, the radius at which the mean enclosed dark matter density is 200 times the critical density. ${ }^{38}$ This implies a relation between $a$ and the scale length $r_{s}$ of the NFW profile. The latter is often given in terms of the concentration index $c$, conventionally defined as $c=r_{200} / r_{s}$. Hence, we adopt the relation ${ }^{36}$ :

$$
a=r_{s} \sqrt{2[\ln (1+\mathrm{c})-\mathrm{c} /(1+\mathrm{c})]} .
$$

For the small dwarf galaxy, we choose a concentration index of $c=25$, which is a value motivated by cosmological simulations ${ }^{39}$ and a spin parameter of 0.09 . Its corresponding radial scalelength $a$ is $0.85 h^{-1} \mathrm{kpc}$. The tidal radius for the small dwarf galaxy computed at the initial distance from the larger dwarf, $30 h^{-1} \mathrm{kpc}$, is $3 h^{-1} \mathrm{kpc}$. This value is almost $30 \%$ of the virial radius and the dark matter mass of the little dwarf galaxy within this radius is $\sim 15$ times larger than the stellar mass disk. The tidal radius changes by time along the orbit and at the pericentric distance is $\sim 1.2 h^{-1} \mathrm{kpc}$.

\section{Disks}

We model the disk component of the stars in each interacting galaxy with a thin exponential surface density profile of scale length $r_{h}$ :

$$
\Sigma_{*}(r)=\frac{M_{*}}{2 \pi r_{h}^{2}} \exp \left(-\mathrm{r} / \mathrm{r}_{\mathrm{h}}\right)
$$

so that the disk mass is $M_{*}=m_{d} M_{\text {tot }}$, where $m_{d}$ is dimensionless and $M_{\text {tot }}$ is the total mass of the galaxy. 


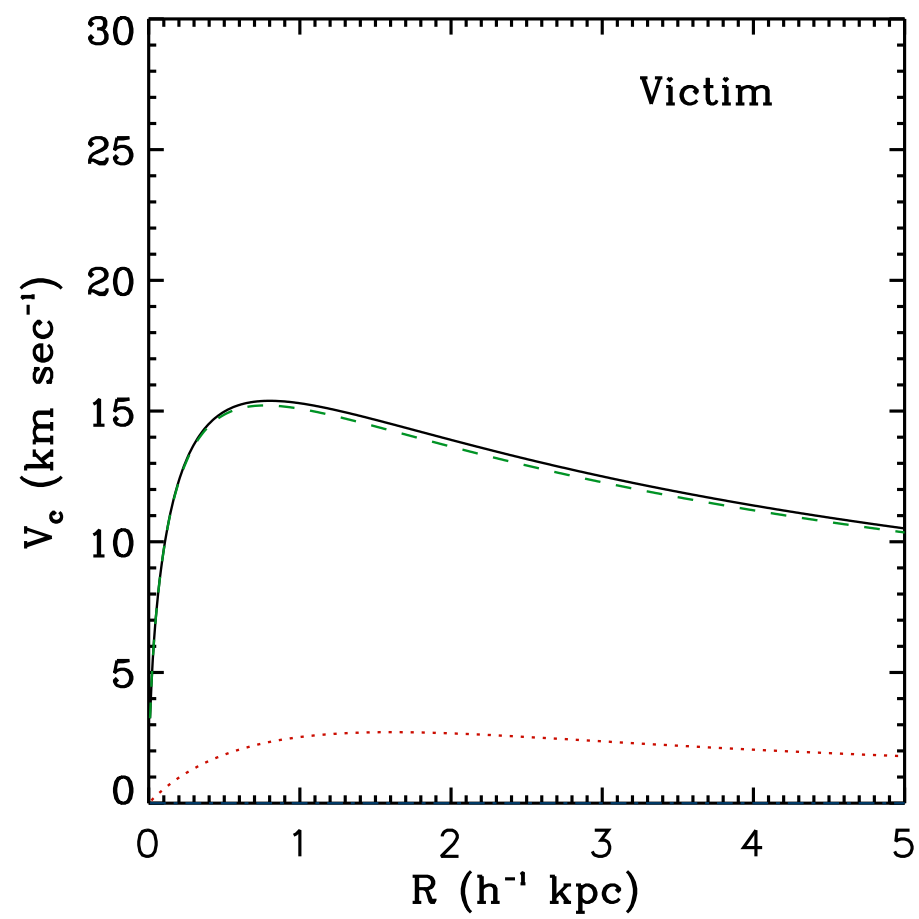

Figure 5. Rotation curve for the small dwarf galaxy model with the following parameters: $v_{200}=10 \mathrm{~km} \mathrm{~s}^{-1}, \mathrm{c}=25$ (the dotted line displays the disk component, the dashed line the dark matter component and the solid line the total rotation curve, dark matter plus disk). The stellar mass disk is assumed to be $5 \%$ of the total dark matter.

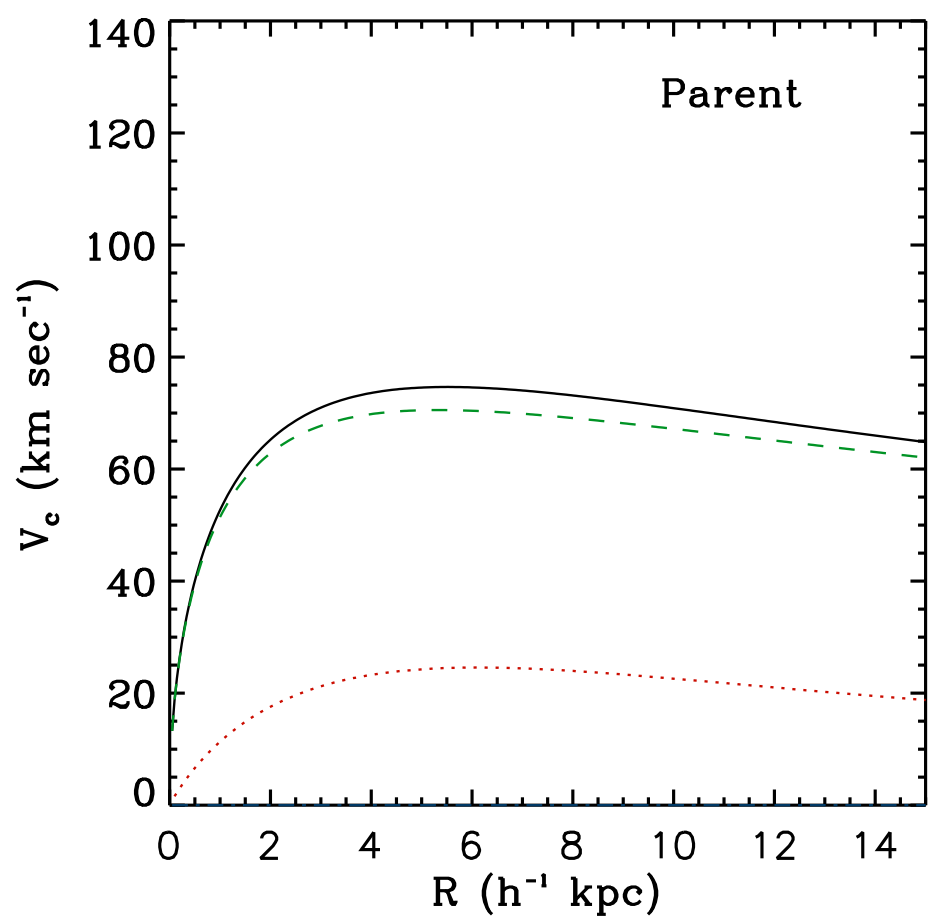

Figure 6. Rotation curve for the modeled larger dwarf galaxy with the following parameters: $v_{200}=50 \mathrm{~km} \mathrm{~s}^{-1}, \mathrm{c}=20$ (line types as above). 


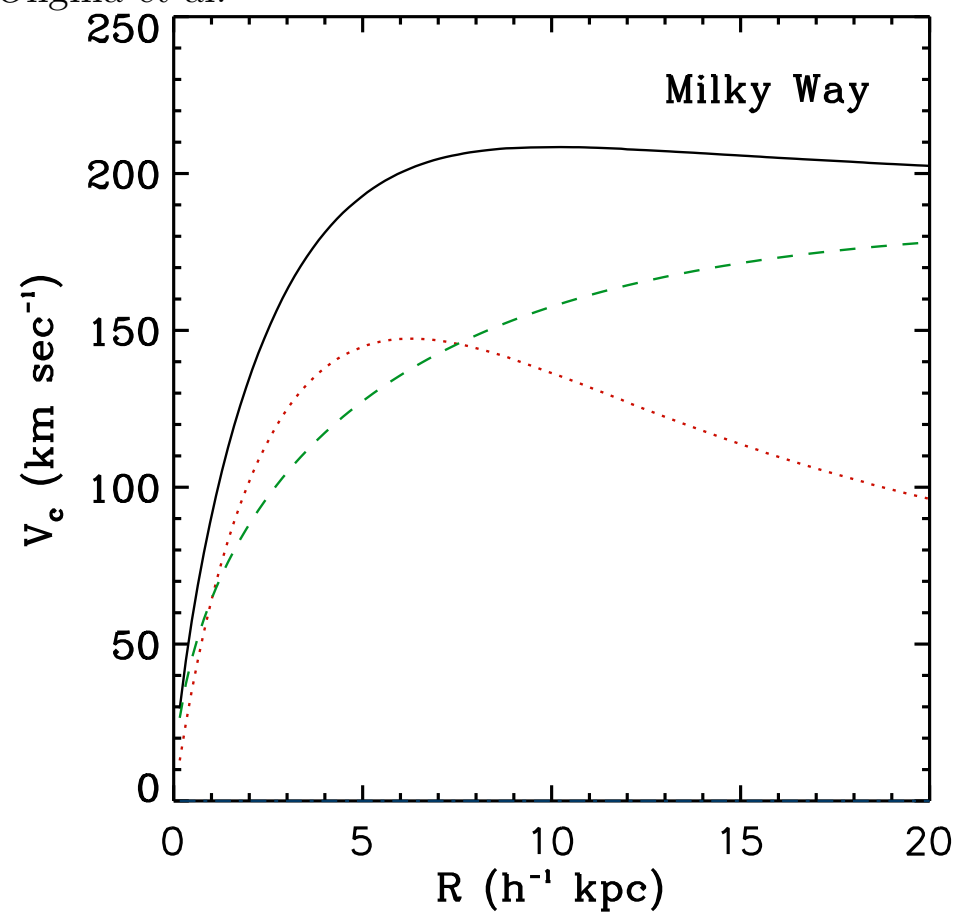

Figure 7. Rotation curve for the Milky Way model with the following parameters: $v_{200}=160 \mathrm{~km} \mathrm{~s}^{-1}, \mathrm{c}=9$ (line types as above).

The characteristic kinematic property of the small dwarf, $v / \sigma$, the ratio between its centrifugal rotational velocity $v$ and its random motions $\sigma$, is measured at the effective radius at each output time. The effective radius $R_{e}$ is defined as the radius enclosing half the projected mass surface density profile, $I\left(R_{e}\right)=I(R) / 2$, with:

$$
I(R)=\int_{0}^{R} \Sigma r d r
$$

The circular velocity of each galaxy is given by:

$$
V_{c}^{2}(r)=\frac{G M_{\mathrm{dm}}(<r)}{r}+\frac{2 G M_{*}}{r_{h}} y^{2} x\left[I_{0}(y) K_{0}(y)-I_{1}(y) K_{1}(y)\right]
$$

Here, $G$ is the gravitational constant, $y=r /\left(2 r_{h}\right)$, and $I_{n}$ and $K_{n}$ are Bessel functions.

We specify the vertical mass distribution of the stars in the disk by giving it the profile of an isothermal sheet with a radially constant scale height $z_{0}$. The $3 \mathrm{D}$ stellar density in the disk is hence given by:

$$
\rho_{*}(r, z)=\frac{M_{*}}{4 \pi z_{0} r_{h}^{2}} \operatorname{sech}^{2}\left(\frac{\mathrm{z}}{2 \mathrm{z}_{0}}\right) \exp \left(-\frac{\mathrm{r}}{\mathrm{r}_{\mathrm{h}}}\right) .
$$

We treat $z_{0}$ as a free parameter that is set by the vertical velocity dispersion of the stars in the disk and fix the velocity distribution of the stars such that this scale height 
is self-consistently maintained in the fully 3D potential of the galaxy model ${ }^{36}$ We adopt $z_{0}=0.1 h^{-1} \mathrm{kpc}$.

Figures 5-7 display the rotation curves for the three galaxy models we employed in our simulations: a small dwarf galaxy, a larger dwarf, and a representation of the Milky Way. The total mass of each galaxy is given in terms of a virial velocity $v_{200}$. We set the total mass as $M_{\text {tot }}=v_{200}^{3} /\left(10 G H_{0}\right)$. The small dwarf has a peak velocity of $15 \mathrm{~km} \mathrm{~s}^{-1}$, implying a total galaxy mass of $1.7 \times 10^{8} h^{-1} \mathrm{M}_{\odot},\left(v_{200}=10 \mathrm{~km} \mathrm{~s}^{-1}\right)$. The larger dwarf galaxy has a peak velocity of $75 \mathrm{~km} \mathrm{~s}^{-1}$ corresponding to a mass of $5 \times 10^{10} h^{-1} \mathrm{M}_{\odot},\left(v_{200}=50 \mathrm{~km} \mathrm{~s}^{-1}\right)$, making it comparable to the Large Magellanic Cloud (LMC).

The galaxy model for the Milky Way has a mass of $9.5 \times 10^{11} h^{-1} \mathrm{M}_{\odot}$ implying a virial velocity $v_{200}=160 \mathrm{~km} \mathrm{~s}^{-1}$. The disk mass of each galaxy is specified by $m_{d}=0.05$. We have set up models with low-mass disks (where the stellar disk is included in the dynamics of the simulations but its mass is negligible) and found that the stars exhibit the same response to the gravitational resonance.

We have also set up models for the small dwarf with a slowly rising rotation curve by assuming a shallow dark matter density profile in the inner parts. For this purpose we have introduced the density profile for the dark matter with the following form ${ }^{40}$ :

$$
\rho=\frac{3 \mathrm{M}}{4 \pi} \frac{a}{(r+a)^{4}} .
$$

This profile belongs to the same family of density profiles as the Hernquist model, but it has a core. The isotropic distribution function for the energy is given by ${ }^{40}$ :

$$
f(\epsilon)=\frac{3 \mathrm{M}}{2 \pi^{3}(G \mathrm{Ma})^{3 / 2}}\left(\sqrt{2 \epsilon} \frac{3-4 \epsilon}{1-2 \epsilon}-3 \operatorname{arcsinh} \sqrt{\frac{2 \epsilon}{1-2 \epsilon}}\right)
$$

We found that this mass distribution is more susceptible to tidal tail formation in agreement with previous works. ${ }^{41}$ In this specific case, $90 \%$ of the stars are preferentially

removed by resonant stripping, resulting in the formation of a dwarf galaxy with a luminous to dark matter ratio a factor of two higher than our previous calculations (see Figure 8).

\section{$5 \quad$ Numerical Tests}

N-body simulations are subject to two-body relaxation effects. These may be particularly severe when several components with different mass particles are present. Massive halo particles may heat up the disk even in the absence of any external perturbation by colliding with lighter stellar particles. If simulations have poor mass resolution, this numerical effect may heat a disk, thickening it with time, and thereby artificially transforming it into a spheroid. The models presented here have a total of $10^{6}$ particles to represent the dark halos and 200,000 stellar particles in the disks. As a simple test, we have evolved the individual galaxies in isolation for many billion years to see whether the discs remain thin 


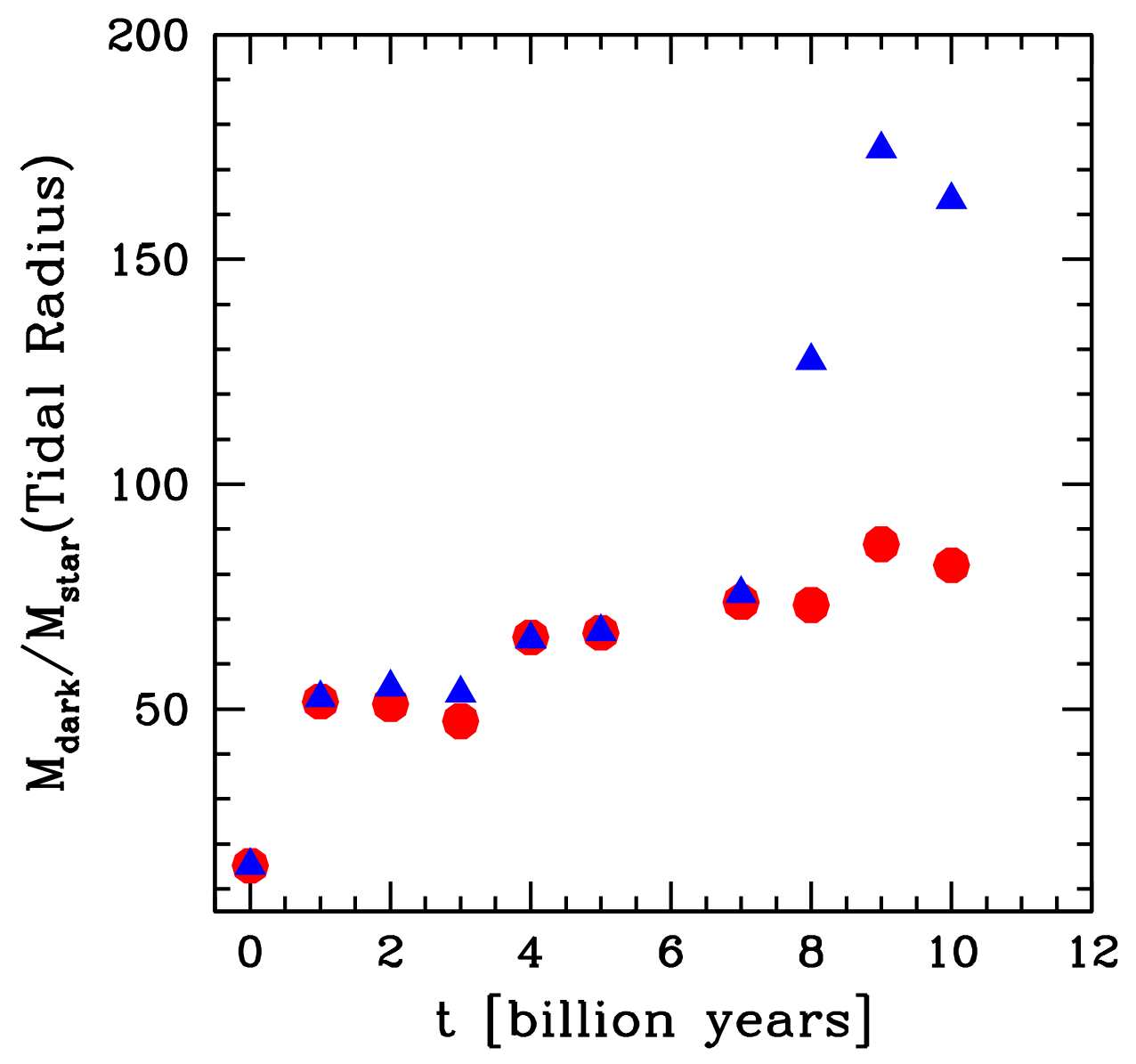

Figure 8. The dark-to-luminous mass ratio $\left(\mathrm{M}_{\mathrm{dm}} / \mathrm{M}_{\text {star }}\right)$ computed at the tidal radius of the smaller dwarf under resonant stripping in a prograde orbit assuming a shallow core for its dark matter density profile as function of time (blue triangles) as compared to a run where a cuspy central dark density profile is assumed (filled red circles). 


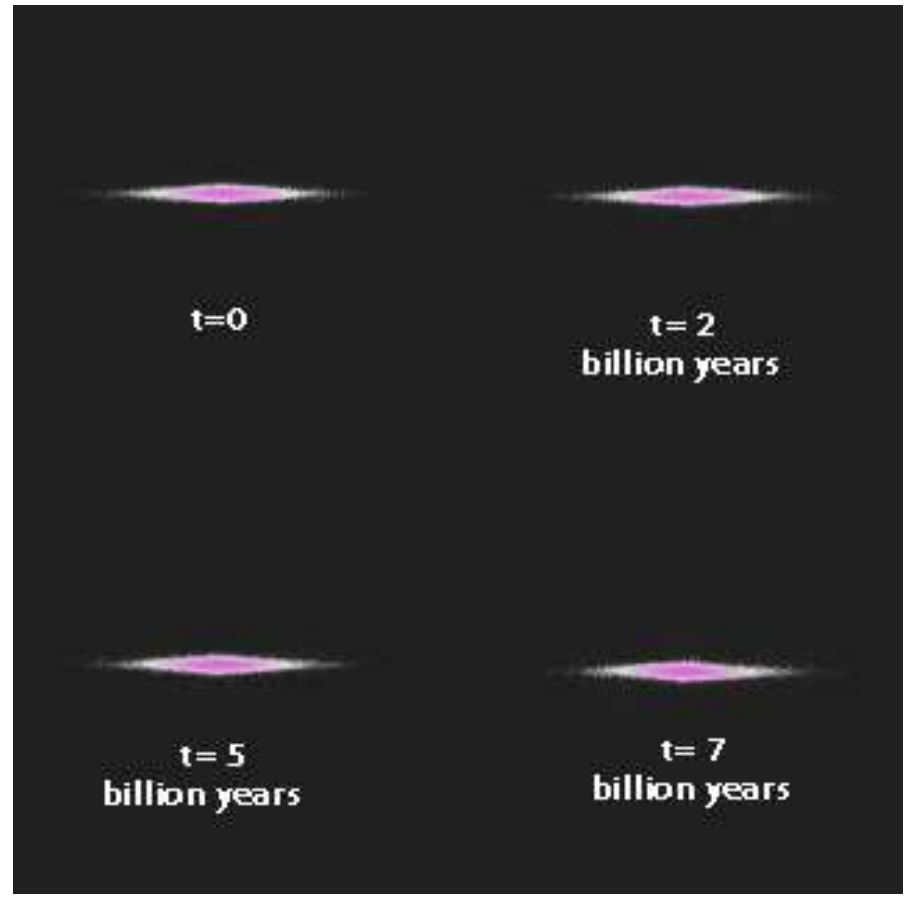

Figure 9. Evolution in time of the disk of the small galaxy simulated in isolation without any external gravitational perturbation. The disk is displayed edge-on at the initial time and after 2, 5 and 7 billion years. The panels are $30 \mathrm{kpc}$ on a side.

or become thicker because of numerical heating. Figure 9 shows that the disk of the small dwarf in isolation (seen edge-on) is nearly static over the course of 7 billion years, indicating that our models are not significantly affected by numerical heating.

\section{Resonant Response on Prograde and Retrograde Orbits}

In our simulations of a small dwarf interacting with a larger dwarf, we place the galaxies on highly eccentric orbits (eccentricity $\mathrm{e}=0.8$ ) with a pericentric distance of $R_{\text {peri }}=5 h^{-1}$ $\mathrm{kpc}$ and an apocentric distance $R_{\text {apo }}=45 h^{-1} \mathrm{kpc}$. These values are likely characteristic of interactions between dwarf galaxies in the small group environments we are simulating. The disks for prograde orbits are inclined with respect to the orbital plane by 30 and 60 degrees. We have also analyzed a coplanar configuration and the results are similar, meaning that the choice of inclination is not critical to these results. For the simulations of a small dwarf interacting with the Milky Way, the galaxies are placed on eccentric orbits $(\mathrm{e}=0.8)$ with $R_{\text {peri }}=10 h^{-1} \mathrm{kpc}$ and $R_{\mathrm{apo}}=100 h^{-1} \mathrm{kpc}$, and the disks are inclined with respect to the orbital plane by the same angles as for the dwarf interactions. In contrast with heating mechanisms like tidal shocking or stripping, the resonance that causes the stripping depends less on the specific choice of the pericentric distances but rather on the combination of the internal structure of the small dwarf (the victim losing stars) and the orbital parameters 


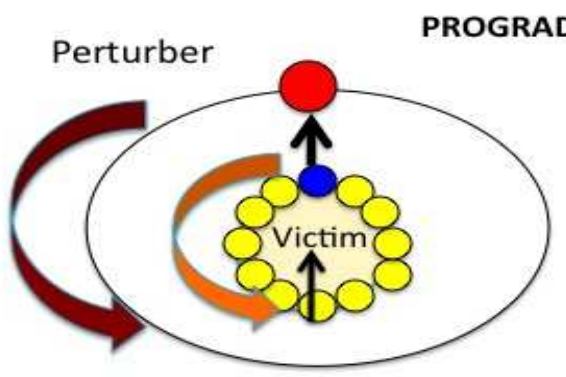

a

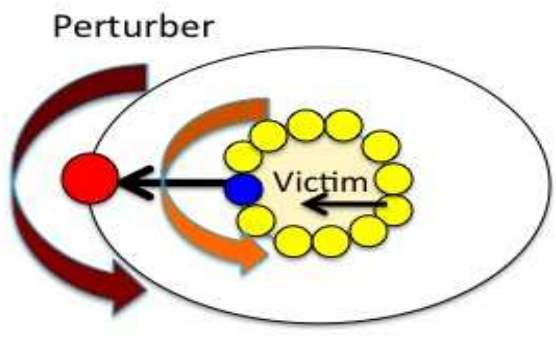

b

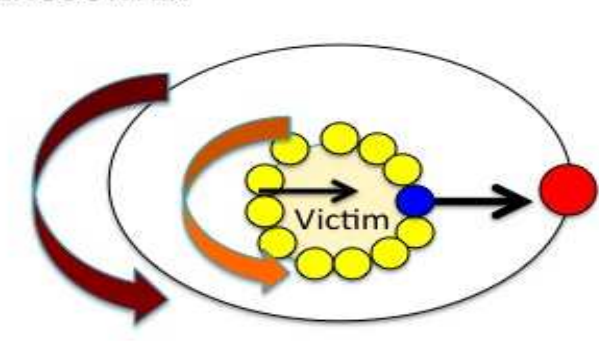

Perturber

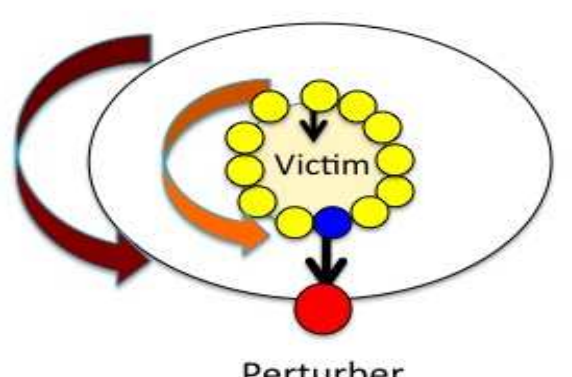

d

Perturber

Figure 10. Resonant stripping response of the small dwarf galaxy (the victim) on prograde orbits under the influence of the gravitational perturbations from the larger dwarf galaxy (perturber). The sequence depicted above is counterclockwise. For prograde encounters the star particles in the victim (drawn as circles), which are rotating in the disk, resonate and are continuously pulled either inward or outward depending on their initial positions in relation to the gravitational perturbation by the larger galaxy. As an example follow the path of the blue circle in the victim.

(see the main text). Full details of the efficiency of this process during encounters between galaxies with different internal structures, including different concentrations and orbital configurations, need to be explored to generalize the applicability of this process.

Resonant stripping is most effective for galaxies colliding on primarily prograde orbits. In this case, as shown schematically in Figure 10-11, stellar particles rotating in the disk of the small galaxy are in resonance and are continuously pulled either inward or outward relative to the center of the disk depending on their initial positions in relation to the gravitational perturbation caused by the larger galaxy (perturber) (Figure 10). For a perfectly retrograde collision, stars in the disk are pulled alternately inward and outward (Figure 11) with little net result. ${ }^{42}$ Because the dark matter particles in the dwarf spheroidal candidate are on random orbits, the net perturbation to the halo tends to average out, greatly suppressing its response. 

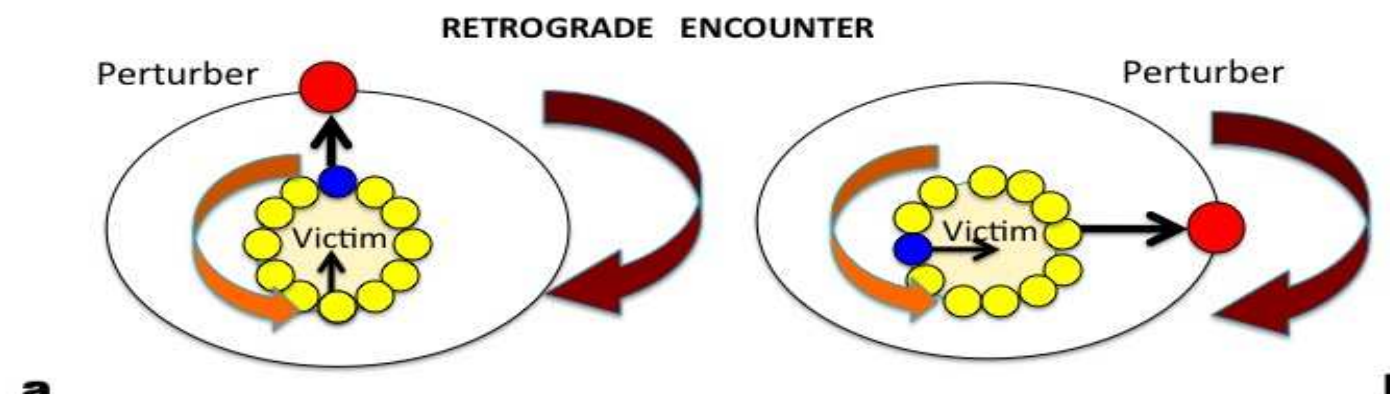

a

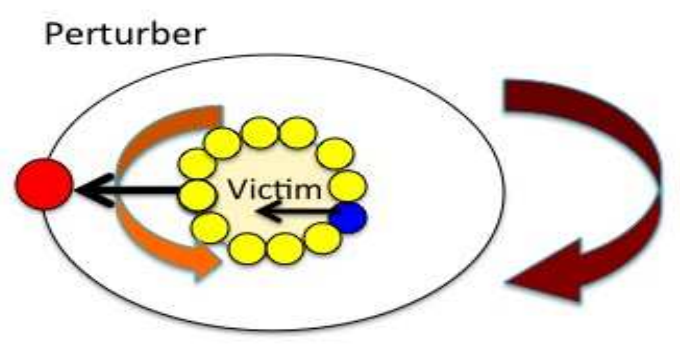

d

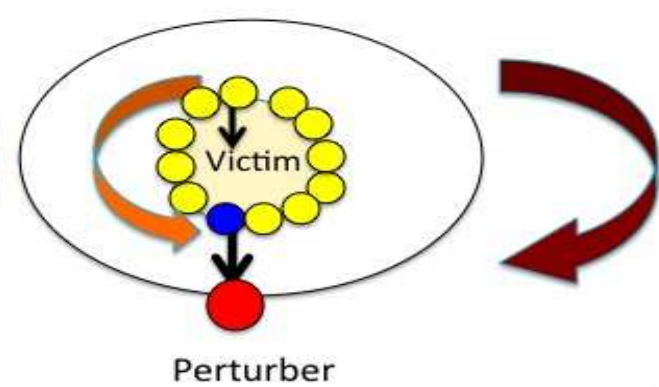

Figure 11. Resonant stripping response of the small dwarf galaxy (the victim) on retrograde orbits under the influence of the gravitational perturbations from the larger dwarf galaxy (perturber). The sequence is clockwise. For a retrograde collision, stars in the disk of the victim are pulled alternately inward and outward (right panel) with little net result. ${ }^{42}$ As an example follow the path of the blue circle in the victim.

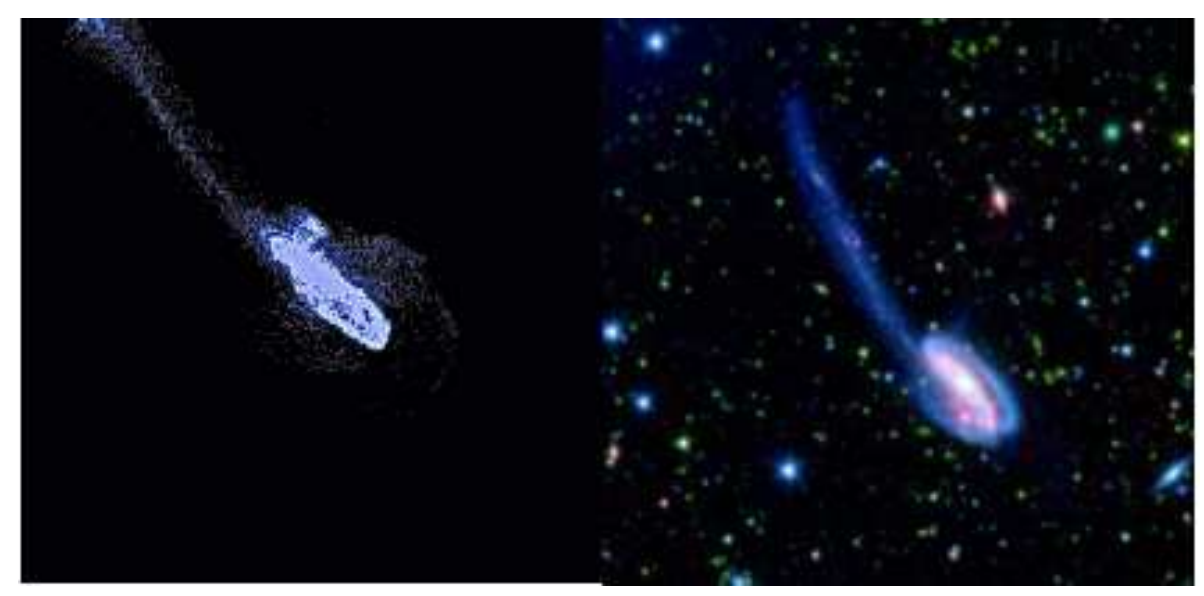

Figure 12. Resonant stripping can produce unusually extended tails (left panel) resembling the long tail observed in the Tadpole galaxy (right panel). 


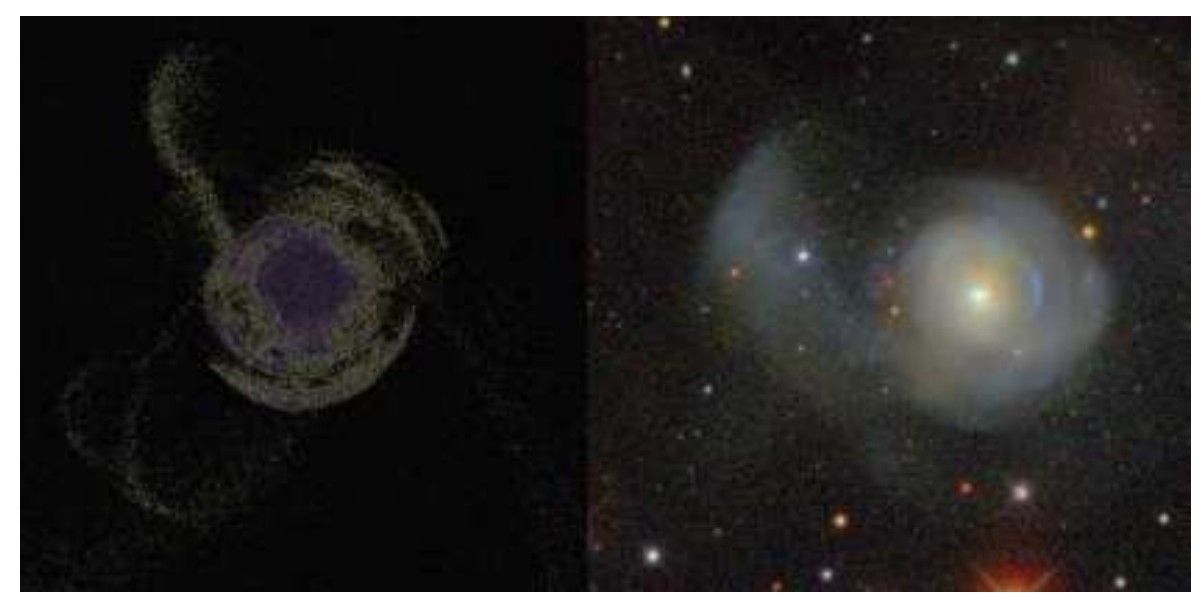

Figure 13. Resonant stripping can produce shells in the larger dwarf galaxy owing to the capture of stars stripped from the encounter with the smaller dwarf. As an example we show an outcome of our simulation after a few billion years (left panel) which resembles the shells of stars observed in NGC 2782 (right panel).

\section{Tails and Shells}

Resonant stripping can pull rotationally supported material like the stars in the dwarfs we simulate, into extended tails and bridges that resemble, e.g., the Tadpole galaxy (see Figure 12), which is believed to have collided with a smaller companion. Our simulations show that the curved tidal tail and shells in this object can be explained by the process of resonant stripping, owing to an interaction with a system 100 times less massive. We argue that unusually long tails can be produced by the fact that star particles rotating faster in the outer part of the disk resonate strongly and are pulled into elongated shapes by the gravitational field of the larger galaxy. The distortion becomes so large that half of the rotating material has been thrown far away from the perturber forming a tidal tail behind the victim with the other half that has been captured by the perturber forming arcs and shells of stars in 7 billion years resembling, e.g., the shells observed in NGC 2782 (see Figure 13).

Received; Accepted .

31. Springel, V., The cosmological simulation code GADGET-2. Mon. Not. R. Astron. Soc., 364, 1105-1134 (2005). 
32. Springel, V. et al., The Aquarius Project: the subhaloes of galactic haloes. Mon. Not. R. Astron. Soc., 391, 1685-1711 (2008).

33. Hernquist, L., Katz, N., TreeSPH: A unification of SPH with the hierarchical tree method. Astrophys. J. Suppl., 70, 419-446 (1989).

34. Hernquist, L., N-body realizations of compound galaxies Astrophys. J. Suppl., 86, 389-400 (1993).

35. Springel, V., Modelling star formation and feedback in simulations of interacting galaxies. Mon. Not. R. Astron. Soc., 312, 859-879 (2000).

36. Springel, V., Di Matteo, T., Hernquist, L. Modelling feedback from stars and black holes in galaxy mergers. Mon. Not. R. Astron. Soc., 361, 776-794 (2005).

37. Hernquist, L. An analytical model for spherical galaxies and bulges. Astrophys. J., 356, 359364 (1990).

38. Navarro, J.F., Frenk, C.S., White, S.D.M. A Universal Density Profile from Hierarchical Clustering. Astrophys. J., 490, 493-508 (1997).

39. Neto, A.F. et al., The statistics of $\Lambda$ CDM halo concentrations. Mon. Not. R. Astron. Soc., 381, 1450-1462 (2007).

40. Dehnen, W. A family of potential-density pairs for spherical galaxies and bulges. Mon. Not. R. Astron. Soc., 265, 250-256 (1993).

41. Dubinsky, J., Mihos, J.C., Hernquist, L. Constraining dark halo potentials with tidal tails. Astrophys. J., 526, 607-622 (1999).

42. Binney, J., Tremaine, S. Galactic Dynamics. Princeton Series in Astrophysics, p. 461 (1987) 